\title{
COHOMOLOGICAL DECOMPOSITION OF COMPACT COMPLEX MANIFOLDS AND HOLOMORPHIC DEFORMATIONS
}

\author{
ADELA LATORRE AND LUIS UGARTE
}

(Communicated by Franc Forstneric)

\begin{abstract}
The main goal of this note is the study of pureness and fullness properties of compact complex manifolds under holomorphic deformations. Firstly, we construct small deformations of pure-and-full complex manifolds along which one of these properties is lost while the other one is preserved. Secondly, we show that the property of being pure-and-full is not closed under holomorphic deformations. In order to do so, we focus on the class of 6 dimensional solvmanifolds endowed with invariant complex structures. In the special case of nilmanifolds, we also give a classification of those invariant complex structures that are both pure and full. In addition, relations of the cohomological decomposition with other metric and complex properties are studied.
\end{abstract}

\section{INTRODUCTION}

Let $(M, J)$ be a compact almost-complex manifold. Denote by $\mathcal{Z}_{J}^{+}(M)$, resp. $\mathcal{Z}_{J}^{-}(M)$, the space of (real) closed 2 -forms that are $J$-invariant, resp. $J$-antiinvariant. Motivated by the Donaldson "tamed to compatible" conjecture [1], $\mathrm{Li}$ and Zhang considered in [19] the subspaces $H_{J}^{+}(M)$ and $H_{J}^{-}(M)$ of the second de Rham cohomology group $H_{\mathrm{dR}}^{2}(M ; \mathbb{R})$ given by

$$
H_{J}^{ \pm}(M):=\left\{\mathbf{a}=[\alpha] \in H_{\mathrm{dR}}^{2}(M ; \mathbb{R}) \mid \alpha \in \mathcal{Z}_{J}^{ \pm}(M)\right\} .
$$

The almost-complex structure $J$ is said to be $\mathcal{C}^{\infty}$-pure if $H_{J}^{+}(M) \cap H_{J}^{-}(M)=\{0\}$, and it is called $\mathcal{C}^{\infty}$-full if $H_{J}^{+}(M)+H_{J}^{-}(M)=H_{\mathrm{dR}}^{2}(M ; \mathbb{R})$. When both properties are satisfied, i.e. the decomposition

$$
H_{\mathrm{dR}}^{2}(M ; \mathbb{R})=H_{J}^{+}(M) \oplus H_{J}^{-}(M)
$$

holds, the almost-complex structure $J$ is called $\mathcal{C}^{\infty}$-pure-and-full.

It turns out [19] that the $J$-compatible cone is an open (possibly empty) convex cone of the $J$-invariant cohomology $H_{J}^{+}(M)$, and the $J$-anti-invariant cohomology $H_{J}^{-}(M)$ measures the difference between the tamed cone and the compatible cone whenever the latter is non-empty and the almost complex structure is $\mathcal{C}^{\infty}$-full. Moreover, Drăghici, Li and Zhang proved in [12] that every compact 4-dimensional almost-complex manifold is $\mathcal{C}^{\infty}$-pure-and-full.

Received by the editors October 6, 2015 and, in revised form, April 5, 2016.

2010 Mathematics Subject Classification. Primary 32G05, 53C15, 53C56, 58A12, 22E25.

Key words and phrases. De Rham cohomology, complex structure, solvmanifold, holomorphic deformation. 
In this paper we focus on the integrable case; that is, $X=(M, J)$ is a compact complex manifold. If the Frölicher spectral sequence of $X$ degenerates at the first step and there is a weight 2 formal Hodge decomposition, then $X$ is $\mathcal{C}^{\infty}$-pureand-full (see [5, 12, 19]). Indeed, in such case the subgroups $H_{J}^{ \pm}(M)$, which we will simply denote by $H^{ \pm}(X)$ in the complex setting, are nothing but the (real) Dolbeault cohomology groups, i.e.

$H^{+}(X)=H_{\bar{\partial}}^{1,1}(X) \cap H_{\mathrm{dR}}^{2}(M ; \mathbb{R}), \quad H^{-}(X)=\left(H_{\bar{\partial}}^{2,0}(X) \oplus H_{\bar{\partial}}^{0,2}(X)\right) \cap H_{\mathrm{dR}}^{2}(M ; \mathbb{R})$.

Hence, compact complex surfaces and compact complex manifolds satisfying the $\partial \bar{\partial}$ Lemma [10] (in particular, compact Kähler manifolds) have the $\mathcal{C}^{\infty}$-pure-and-full property, as well as any sufficiently small holomorphic deformation of a compact $\partial \bar{\partial}$-manifold [7, 27]. In contrast, the Iwasawa manifold is $\mathcal{C}^{\infty}$-pure-and-full, but there are small deformations which are not $\mathcal{C}^{\infty}$-pure-and-full. Moreover, along any deformation of the Iwasawa manifold the $\mathcal{C}^{\infty}$-pure property fails if and only if the $\mathcal{C}^{\infty}$-full property does [5. The first aim of this paper is to construct small deformations of $\mathcal{C}^{\infty}$-pure-and-full compact complex manifolds along which one of the properties is lost while the other one is preserved.

Our second aim is to analyze the behaviour of the deformation limit $X_{0}$ of an analytic family of $\mathcal{C}^{\infty}$-pure-and-full compact complex manifolds $X_{t}$. This is mainly motivated by an interest in studying the deformation limits of cohomological and/or metrical properties of compact complex manifolds (see [23] for more details). In [9] it is proved that the strongly Gauduchon property is not closed. Furthermore, the central limit of an analytic family of compact balanced manifolds may not have any strongly Gauduchon metric. In [15] (see also [4]) one can find an analytic family of compact balanced $\partial \bar{\partial}$-manifolds $X_{t}$ such that the central limit $X_{0}$ neither satisfies the $\partial \bar{\partial}$-Lemma nor admits balanced metrics. Here we show that the $\mathcal{C}^{\infty}$-pure-andfull property is not deformation closed.

A Hermitian-symplectic structure on a compact complex manifold $X$ is a taming symplectic form (see [17] in relation to other geometric structures in the almostcomplex setting). In complex dimension 2, if $X$ has a Hermitian-symplectic structure, then it admits a Kähler metric [19,25]. Streets and Tian posed in [25] the problem of finding compact Hermitian-symplectic manifolds not admitting Kähler metrics. Up to now, the related results in the literature suggest that Hermitiansymplectic structures do not exist on non-Kähler manifolds. Thus, one might think that any compact Hermitian-symplectic manifold should satisfy the $\mathcal{C}^{\infty}$-pure-andfull property, although this has not yet been proved. In this context, it seems natural to ask to what extent the existence of a special non-Kähler Hermitian metric has an influence on the $\mathcal{C}^{\infty}$-pure-and-full property. The third aim of this paper is to show that the latter property is unrelated to the SKT, locally conformal Kähler, balanced, and strongly Gauduchon properties.

The paper is structured as follows. First, let us remark that the class of compact complex manifolds that we consider in order to prove the results mentioned above is that of 6-dimensional solvmanifolds endowed with invariant complex structures $J$. Recall that a solvmanifold (resp. nilmanifold) is a compact manifold $M=\Gamma \backslash G$ obtained as a quotient of a simply-connected solvable (resp. nilpotent) Lie group $G$ by a lattice $\Gamma$. An invariant complex structure $J$ on $M$ is a complex structure coming from a left-invariant complex structure on $G$. In Section 2 we prove that in the class of 6-nilmanifolds there exist, up to isomorphism, exactly four invariant complex structures satisfying the $\mathcal{C}^{\infty}$-pure-and-full property, apart from the torus. 
Moreover, two of them live on the nilmanifold underlying the Iwasawa manifold and the other two on "its 3-step analogue". Except for the Iwasawa manifold, the other three complex structures seem to provide new examples of $\mathcal{C}^{\infty}$-pure-and-full compact complex manifolds.

In Section 3 we study the behaviour of holomorphic deformations of $\mathcal{C}^{\infty}$-pureand-full manifolds. Let $h_{J}^{ \pm}(M)$ denote the dimension of $H_{J}^{ \pm}(M)$. In [13, Drăghici, $\mathrm{Li}$ and Zhang prove that for any curve of almost-complex structures $J_{t}$ on a 4dimensional compact manifold $M^{4}$, the dimension $h_{J_{t}}^{+}\left(M^{4}\right)$ is a lower-semicontinuous function in $t$, whereas $h_{J_{t}}^{-}\left(M^{4}\right)$ is upper-semi-continuous. Angella and Tomassini show in [6] that these properties are no longer true in dimension $\geq 6$, by means of two explicit families of curves $J_{t}$. Since such families $J_{t}$ are not $\mathcal{C}^{\infty}$ pure for $t \neq 0$, they wonder if "more fulfilling counterexamples" could be found. In Proposition 3.1 we give a holomorphic deformation $X_{t}$ of a $\mathcal{C}^{\infty}$-pure-and-full compact complex manifold $X_{0}$ of complex dimension 3 such that $X_{t}$ is $\mathcal{C}^{\infty}$-pure for every $t$, but $h^{+}\left(X_{0}\right)>h^{+}\left(X_{t}\right)$ for any $t \neq 0$. Proposition 3.3 provides another family $X_{t}$ in complex dimension 3 satisfying $h^{-}\left(X_{0}\right)<h^{-}\left(X_{t}\right)$ for any $t \neq 0$. In the last part of the section we revisit the small deformations of the Iwasawa manifold to show that the dimension of $H^{+}$remains constant along them (see Proposition 3.4). Moreover, Example 3.5 contains a deformation $X_{t}$ of the Iwasawa manifold along which $H^{-}$changes drastically from satisfying $H^{+}\left(X_{0}\right) \oplus H^{-}\left(X_{0}\right)=H_{\mathrm{dR}}^{2}$ to being completely contained in the subspace $H^{+}$, i.e. $\{0\} \neq H^{-}\left(X_{t}\right) \varsubsetneqq H^{+}\left(X_{t}\right) \varsubsetneqq H_{\mathrm{dR}}^{2}$ for any $t \neq 0$.

In Section 4 we prove that the $\mathcal{C}^{\infty}$-pure-and-full property is not closed under holomorphic deformations. Using the study of 6-dimensional solvmanifolds with holomorphically trivial canonical bundle [15], we find an analytic family of compact complex manifolds $X_{t}$ such that $X_{t}$ is $\mathcal{C}^{\infty}$-pure-and-full for every $t \neq 0$, but $X_{0}$ is neither $\mathcal{C}^{\infty}$-pure nor $\mathcal{C}^{\infty}$-full (see Theorem 4.1). Therefore, for compact complex manifolds, the properties of being $\mathcal{C}^{\infty}$-pure-and-full, being $\mathcal{C}^{\infty}$-pure, and being $\mathcal{C}^{\infty}$ full are not closed under holomorphic deformations.

Finally, Section 5 is devoted to illustrating that the $\mathcal{C}^{\infty}$-pure-and-full property is unrelated to the SKT, locally conformal Kähler, balanced, and strongly Gauduchon properties (see Corollary 5.2). With respect to the Frölicher spectral sequence, Proposition 5.3 shows the existence of a compact complex manifold $X$ with $E_{1}(X) \cong$ $E_{\infty}(X)$ whose Hodge numbers satisfy the symmetry $h_{\bar{\partial}}^{p, q}(X)=h_{\bar{\partial}}^{q, p}(X)$, which is neither $\mathcal{C}^{\infty}$-pure nor $\mathcal{C}^{\infty}$-full. In the case of 6 -dimensional nilmanifolds $M$, we also give a relation between the invariant complex structures $J$ satisfying the $\mathcal{C}^{\infty}$ pure-and-full property and the maximal values of the complex invariant $\Delta(M, J)$ introduced by Angella and Tomassini in [7] (see Propositions 5.4 and [5.5).

\section{Classification of $\mathcal{C}^{\infty}$-PURE-AND-FUll COMPlex Nilmanifolds}

Let $M=\Gamma \backslash G$ be a nilmanifold of dimension $2 n$ and $J$ an invariant complex structure on $M$. Let $\mathfrak{g}$ be the Lie algebra of $G$ and let $\mathfrak{g}^{1,0}$ be the elements in $\mathfrak{g}_{\mathbb{C}}^{*}$ of bidegree $(1,0)$ with respect to $J$. By Salamon's characterization of the integrability condition of complex structures on nilpotent Lie algebras [24], $\mathfrak{g}^{1,0}$ has a basis $\left\{\omega^{k}\right\}_{k=1}^{n}$ such that $d \omega^{1}=0$ and

$$
d \omega^{k} \in \mathrm{I}\left(\omega^{1}, \ldots, \omega^{k-1}\right), \quad \text { for } k=2, \ldots, n,
$$


where $\mathrm{I}\left(\omega^{1}, \ldots, \omega^{k-1}\right)$ is the ideal in the exterior algebra $\Lambda^{*}\left(\mathfrak{g}_{\mathbb{C}}^{*}\right)$ generated by $\left\{\omega^{1}, \ldots, \omega^{k-1}\right\}$. From now on, $\omega^{k} \wedge \omega^{l}$ and $\omega^{k} \wedge \omega^{\bar{l}}$ will be respectively denoted by $\omega^{k l}$ and $\omega^{k \bar{l}}$.

Special attention should be drawn to Abelian structures, which are those satisfying the condition $[J x, J y]=[x, y]$, for all $x, y \in \mathfrak{g}$. The reason for the interest in these complex structures in relation to the cohomological decomposition is clear by the following result.

Lemma 2.1. Let $J$ be an Abelian complex structure on a $2 n$-dimensional nilmanifold $M$. Then, $X=(M, J)$ is always $\mathcal{C}^{\infty}$-pure.

Proof. We can use a Nomizu type result for the subgroups $H^{+}(X)$ and $H^{-}(X)$ (see [8, Theorem 5.4] and [16. Theorem 3.4]) to reduce the proof to the level of invariant forms on $M$. Let $\mathbf{a} \in H^{+}(X) \cap H^{-}(X)$, and let $\beta \in \mathcal{Z}_{J}^{+}\left(\mathfrak{g}^{*}\right)$ and $\gamma \in \mathcal{Z}_{J}^{-}\left(\mathfrak{g}^{*}\right)$ be real closed invariant forms such that $[\beta]=\mathbf{a}=[\gamma]$. Then, there exists an element $\alpha \in \bigwedge^{1}\left(\mathfrak{g}^{*}\right)$ satisfying $d \alpha=\beta-\gamma$. Using the bidegree decomposition of $\alpha$ as $\alpha=\alpha_{1,0}+\overline{\alpha_{1,0}}$, we get

$$
d \alpha=\left(\bar{\partial} \alpha_{1,0}+\partial \overline{\alpha_{1,0}}\right)+\left(\partial \alpha_{1,0}+\bar{\partial} \overline{\alpha_{1,0}}\right)=\beta-\gamma,
$$

which implies that $\beta=\bar{\partial} \alpha_{1,0}+\partial \overline{\alpha_{1,0}}$ and $\gamma=-\left(\partial \alpha_{1,0}+\bar{\partial} \overline{\alpha_{1,0}}\right)$. However, when $J$ is Abelian the Lie algebra differential $d$ satisfies $d\left(\bigwedge^{1,0}\left(\mathfrak{g}^{*}\right)\right) \subset \bigwedge^{1,1}\left(\mathfrak{g}^{*}\right)$; thus $\partial \alpha_{1,0}=0$ and so $\gamma=0$. We conclude that the form $\beta=d \alpha$ is exact, i.e. $\mathbf{a}=[\beta]=\mathbf{0}$, and therefore $H^{+}(X) \cap H^{-}(X)=\{\mathbf{0}\}$.

Remark 2.2. Lemma 2.1 can be extended with the same proof to the bigger class of solvmanifolds for which the natural map $\iota: H^{2}(\mathfrak{g}) \hookrightarrow H_{\mathrm{dR}}^{2}(M ; \mathbb{R})$ is an isomorphism. That is to say, any Abelian complex structure on this type of solvmanifolds is $\mathcal{C}^{\infty}$ pure.

Let us now focus on the case of 6-dimensional nilmanifolds endowed with invariant complex structures. In the following result we prove that there exist precisely four complex structures satisfying the $\mathcal{C}^{\infty}$-pure-and-full property. Two of them live on the nilmanifold underlying the Iwasawa manifold and the other two on "its 3step analogue". In more detail, the structure equations of these two nilmanifolds, which we will respectively denote by $\mathcal{N}_{0}$ and $\mathcal{N}_{1}$, are given by

$$
\mathcal{N}_{\epsilon}: \quad d e^{1}=d e^{2}=d e^{3}=0, \quad d e^{4}=\epsilon e^{12}, \quad d e^{5}=e^{13}-e^{24}, \quad d e^{6}=e^{14}+e^{23},
$$

where $\epsilon \in\{0,1\}$. Notice that $\mathcal{N}_{0}$ corresponds to $\mathfrak{h}_{5}$ and $\mathcal{N}_{1}$ to $\mathfrak{h}_{15}$ in the notation of 9 .

Theorem 2.3. Let $X=(M, J)$ be a 6-dimensional nilmanifold $M$, not a torus, endowed with an invariant complex structure $J$. Then, $X$ is $\mathcal{C}^{\infty}$-pure-and-full if and only if $X=\left(\mathcal{N}_{\epsilon}, \mathcal{I}_{\epsilon}^{\rho}\right)$, where $\epsilon, \rho \in\{0,1\}$, and

$$
\mathcal{I}_{\epsilon}^{\rho}: \quad d \omega^{1}=0, \quad d \omega^{2}=\epsilon \omega^{1 \overline{1}}, \quad d \omega^{3}=\rho \omega^{12}+(1-\rho) \omega^{1 \overline{2}} .
$$

Proof. First recall that there exist two complex-parallelizable nilmanifolds, defined by the equations

$$
d \omega^{1}=d \omega^{2}=0, \quad d \omega^{3}=\rho \omega^{12},
$$

where $\rho \in\{0,1\}$. One is the torus $(\rho=0)$, and the other one is the Iwasawa manifold $(\rho=1)$. The latter precisely corresponds to $\mathcal{I}_{0}^{1}$ in the statement of the theorem. It is well-known that these complex structures are $\mathcal{C}^{\infty}$-pure-and-full [5,16]. 
Next we use the description of the remaining invariant complex structures $J$ on 6-dimensional nilmanifolds obtained in [9], where they are divided into three families:

(I) $d \omega^{1}=d \omega^{2}=0, \quad d \omega^{3}=\rho \omega^{12}+\omega^{1 \overline{1}}+\lambda \omega^{1 \overline{2}}+D \omega^{2 \overline{2}} \quad\left(\rho \in\{0,1\}, \lambda \in \mathbb{R}^{\geq 0}\right.$ and $D \in \mathbb{C}$ with $\mathfrak{I m} D \geq 0$ );

(II) $d \omega^{1}=0, d \omega^{2}=\omega^{1 \overline{1}}, d \omega^{3}=\rho \omega^{12}+B \omega^{1 \overline{2}}+c \omega^{2 \overline{1}} \quad\left(\rho \in\{0,1\}, B \in \mathbb{C}, c \in \mathbb{R}^{\geq 0}\right.$, with $(\rho, B, c) \neq(0,0,0))$;

$$
d \omega^{1}=0, \quad d \omega^{2}=\omega^{13}+\omega^{1 \overline{3}}, \quad d \omega^{3}=\varepsilon i \omega^{1 \overline{1}} \pm i \omega^{1 \overline{2}} \mp i \omega^{2 \overline{1}} \quad(\varepsilon \in\{0,1\}) .
$$

Notice that the Abelian structures correspond to $\rho=0$ in the families (I) and (II).

In what follows, as a matter of notation, $\delta_{\text {expression }}=1$ if expression $=0$, and 0 if expression $\neq 0$. We will study the $\mathcal{C}^{\infty}$-pure-and-full property for each one of these families.

Let $J$ be in the family (I). If $\rho=1$, then the following relation in de Rham cohomology holds:

$$
\left[i\left(\omega^{12}-\omega^{\overline{1} \overline{2}}\right)\right]=-2\left[i \omega^{1 \overline{1}}\right]-\lambda\left[i\left(\omega^{1 \overline{2}}+\omega^{2 \overline{1}}\right)\right]-2 \mathfrak{R e} D\left[i \omega^{2 \overline{2}}\right] .
$$

Notice that the cohomology class on the left hand side of this equality belongs to $H_{J}^{+}$and the class on the right hand side to $H_{J}^{-}$. Thus, we have that $H_{J}^{+} \cap H_{J}^{-} \neq \mathbf{0}$ and $J$ is not $\mathcal{C}^{\infty}$-pure. Hence, for complex structures in the family (I), we are led to consider the case $\rho=0$; i.e. the complex structure is Abelian. By Lemma 2.1 we know that any Abelian structure $J$ is $\mathcal{C}^{\infty}$-pure, so we just need to see when $J$ is $\mathcal{C}^{\infty}$-full. Observe that the pureness of the structure implies that $H_{J}^{+}+H_{J}^{-}$is a direct sum, so the fullness of $J$ is equivalent to the sum $h_{J}^{+}+h_{J}^{-}$being equal to the second Betti number $b_{2}$.

According to the classification in 9, for every Abelian complex structure in the family (I) we can take $\lambda$ to be 0 or 1 , and a direct computation shows that

$$
\begin{aligned}
& H_{J}^{+}=\left\langle\left[i\left(\omega^{1 \overline{2}}+\omega^{2 \overline{1}}\right)\right], \delta_{\lambda}\left[\omega^{1 \overline{2}}-\omega^{2 \overline{1}}\right],\left(\delta_{\lambda-1}+\delta_{\lambda} \delta_{\mathfrak{I} \mathfrak{m} D}\right)\left[i \omega^{2 \overline{2}}\right],\right. \\
&\left.\delta_{D}\left[\omega^{1 \overline{3}}-\omega^{3 \overline{1}}+\lambda\left(\omega^{2 \overline{3}}-\omega^{3 \overline{2}}\right)\right], \delta_{D}\left[i\left(\omega^{1 \overline{3}}+\omega^{3 \overline{1}}\right)+i \lambda\left(\omega^{2 \overline{3}}+\omega^{3 \overline{2}}\right)\right]\right\rangle, \\
& H_{J}^{-}=\left\langle\left[\omega^{12}+\omega^{\overline{1} \overline{2}}\right],\left[i\left(\omega^{12}-\omega^{\overline{1} \overline{2}}\right)\right], \delta_{D}\left[\omega^{13}+\omega^{\overline{1} \overline{3}}\right], \delta_{D}\left[i\left(\omega^{13}-\omega^{\overline{1} \overline{3}}\right)\right]\right\rangle .
\end{aligned}
$$

Taking into account that $b_{2} \geq 8$ for any of the underlying Lie algebras (see 9 and [24]), it is clear that the complex structures with $D \neq 0$ are not $\mathcal{C}^{\infty}$-full. If $D=0$ and $\lambda=0$, the underlying algebra is $(0,0,0,0,0,12)$, but its second Betti number equals 11. Thus, this complex structure is not $\mathcal{C}^{\infty}$-full. However, if $D=0$ and $\lambda=1$, then $J$ satisfies $h_{J}^{+}+h_{J}^{-}=4+4=8=b_{2}$, and $J$ is $\mathcal{C}^{\infty}$-pure-and-full (note that $J$ is equivalent to the complex structure $\mathcal{I}_{0}^{0}$ in the statement of the theorem).

Let $J$ be a complex structure in the family (II). If $\rho=1$ and $B=c=0$, then the second de Rham cohomology group is given by

$H_{\mathrm{dR}}^{2}(M ; \mathbb{R})=\left\langle\left[\omega^{1 \overline{2}}-\omega^{2 \overline{1}}\right],\left[i\left(\omega^{1 \overline{2}}+\omega^{2 \overline{1}}\right)\right],\left[i\left(\omega^{1 \overline{3}}+\omega^{2 \overline{2}}+\omega^{3 \overline{1}}\right)\right],\left[\omega^{13}+\omega^{\overline{1} \overline{3}}\right],\left[i\left(\omega^{13}-\omega^{\overline{1} \overline{3}}\right)\right]\right\rangle$.

This complex structure, which is precisely $\mathcal{I}_{1}^{1}$, is clearly $\mathcal{C}^{\infty}$-pure-and-full. 
Let us now suppose that $\rho=1$ and $(B, c) \neq(0,0)$. Two cases can be distinguished:

- If $I \mathfrak{m} B \neq 0$, then the following relation in de Rham cohomology holds:

$$
\left[i\left(\omega^{12}-\omega^{\overline{1} \overline{2}}\right)\right]=\mathfrak{I m} B\left[\omega^{1 \overline{2}}-\omega^{2 \overline{1}}\right]-(\mathfrak{R e} B+c)\left[i\left(\omega^{1 \overline{2}}+\omega^{2 \overline{1}}\right)\right],
$$

which implies that the structure is not $\mathcal{C}^{\infty}$-pure;

- If $\mathfrak{I m} B=0$ and $c \neq-B$, then we have the same relation as above, $\left[i\left(\omega^{12}-\omega^{\overline{1} \overline{2}}\right)\right]=-(B+c)\left[i\left(\omega^{1 \overline{2}}+\omega^{2 \overline{1}}\right)\right]$, so it is not $\mathcal{C}^{\infty}$-pure. If $\mathfrak{I m} B=0$ and $c=-B \neq 0$, then the relation $\left[\omega^{12}+\omega^{\overline{1} \overline{2}}\right]=-2 B\left[\omega^{1 \overline{2}}-\omega^{2 \overline{1}}\right]$ is satisfied, so again it is not $\mathcal{C}^{\infty}$-pure.

Hence, for complex structures in the family (II), it remains to study the case $\rho=0$; i.e. the complex structure $J$ is Abelian. By Lemma 2.1 we know that $J$ is $\mathcal{C}^{\infty}$-pure, so it suffices to see when the complex structure is $\mathcal{C}^{\infty}$-full. We proceed as in the case of family (I), now taking into account that we can suppose $B=0$ or 1 due to the choice of $\rho=0$ (see [9] for more details). By direct calculation we get

$$
\begin{aligned}
& H_{J}^{+}=\left\langle\delta_{B}\left[\omega^{1 \overline{3}}-\omega^{3 \overline{1}}\right], \delta_{B}\left[i\left(\omega^{1 \overline{3}}+\omega^{3 \overline{1}}\right)\right], \delta_{B-1}\left[i\left(\omega^{1 \overline{3}}+\omega^{2 \overline{2}}+\omega^{3 \overline{1}}\right)\right], \delta_{B-1} \delta_{c-1}\left[\omega^{1 \overline{2}}-\omega^{2 \overline{1}}\right]\right\rangle, \\
& H_{J}^{-}=\left\langle\left[\omega^{12}+\omega^{\overline{1} \overline{2}}\right],\left[i\left(\omega^{12}-\omega^{\overline{1} \overline{2}}\right)\right], \delta_{c}\left[\omega^{13}+\omega^{\overline{1} \overline{3}}\right], \delta_{c}\left[i\left(\omega^{13}-\omega^{\overline{1} \overline{3}}\right)\right]\right\rangle .
\end{aligned}
$$

Since the considered complex structures satisfy $h_{J}^{+} \leq 2$ and the underlying algebras have $b_{2} \geq 5$ (see 9 and [24]), it is clear that $c \neq 0$ implies non- $\mathcal{C}^{\infty}$-fullness.

If $c=0$, then we must have $B=1$, and thus $h_{J}^{+}+h_{J}^{-}=1+4=5=b_{2}$. Consequently, this complex structure, which is precisely $\mathcal{I}_{1}^{0}$, satisfies the $\mathcal{C}^{\infty}$-pureand-full property.

For complex structures $J$ in the family (III), it is easy to see that

$$
H_{J}^{+}=\left\langle\delta_{\varepsilon}\left[i \omega^{1 \overline{1}}\right]\right\rangle, \quad H_{J}^{-}=\left\langle\left[\omega^{12}+\omega^{\overline{1} \overline{2}}\right],\left[i\left(\omega^{12}-\omega^{\overline{1} \overline{2}}\right)\right]\right\rangle .
$$

The absence of relations between the de Rham cohomology classes allows to conclude that these structures are always $\mathcal{C}^{\infty}$-pure. As the underlying Lie algebras have second Betti number $b_{2} \geq 4$ (see 9] and 24]), it is clear that they are non$\mathcal{C}^{\infty}$-full.

Finally, the Lie algebras underlying the complex structures $\mathcal{I}_{\epsilon}^{\rho}$ are obtained as follows. Take the real basis $\left\{e^{1}, \ldots, e^{6}\right\}$ given by

$$
\omega^{1}=e^{1}+i e^{2}, \quad \omega^{2}=-2(2 \rho-1) e^{3}-2 i e^{4}, \quad \omega^{3}=-2(2 \rho-1)\left(e^{5}+i e^{6}\right) .
$$

A direct calculation shows that

$d e^{1}=d e^{2}=d e^{3}=0, \quad d e^{4}=\varepsilon e^{1} \wedge e^{2}, \quad d e^{5}=e^{1} \wedge e^{3}+e^{4} \wedge e^{2}, \quad d e^{6}=e^{1} \wedge e^{4}+e^{2} \wedge e^{3}$,

so the underlying Lie algebra only depends on $\varepsilon$. In fact, if $\epsilon=0$, then the nilmanifold is the real manifold underlying the Iwasawa manifold (whose Lie algebra is $\left.\mathfrak{h}_{5}=(0,0,0,0,13+42,14+23)\right)$, whereas if $\epsilon=1$, then the underlying Lie algebra is $\mathfrak{h}_{15}=(0,0,0,12,13+42,14+23)$.

As we observed above, the compact complex manifold $\left(\mathcal{N}_{0}, \mathcal{I}_{0}^{1}\right)$ is the well-known Iwasawa manifold. Notice that the complex structures in $\left(\mathcal{N}_{0}, \mathcal{I}_{0}^{0}\right)$ and $\left(\mathcal{N}_{1}, \mathcal{I}_{1}^{0}\right)$ are Abelian. In the next section we construct some holomorphic deformations of the compact complex manifolds $\left(\mathcal{N}_{\epsilon}, \mathcal{I}_{\epsilon}^{\rho}\right)$ with interesting properties with respect to the $\mathcal{C}^{\infty}$-pure-and-full property. 


\section{Holomorphic Deformations of $\mathcal{C}^{\infty}$-PURE-AND-FUlL COMPLEX NILMANIFOLDS}

It is well-known that the property of being $\mathcal{C}^{\infty}$-pure-and-full is not open under holomorphic deformations, the small deformations of the Iwasawa manifold being an example [5]. It is worth observing that in this specific case the $\mathcal{C}^{\infty}$-pure and the $\mathcal{C}^{\infty}$-full properties fail simultaneously, remaining unclear how closely related their behaviours under holomorphic deformation are. In this section we provide examples showing that it is possible to lose $\mathcal{C}^{\infty}$-fullness while preserving $\mathcal{C}^{\infty}$-pureness, but also the converse. These facts allow us to conclude that the previous properties are nonopen in an independent way. In Subsection 3.1 we revisit the small deformations of the Iwasawa manifold to show that $h^{+}$remains constant and to determine a deformation $X_{t}$ along which $H^{-}$changes drastically from $H^{+}\left(X_{0}\right) \oplus H^{-}\left(X_{0}\right)=H_{\mathrm{dR}}^{2}$ to being completely contained in the subspace $H^{+}$, i.e. $H^{-}\left(X_{t}\right) \subset H^{+}\left(X_{t}\right)$ for any $t \neq 0$.

As we reminded before, Drăghici, Li and Zhang prove in 13 that for any curve of almost-complex structures $J_{t}$ on a 4-dimensional compact manifold $M^{4}, h_{J_{t}}^{+}\left(M^{4}\right)$ is a lower-semi-continuous function in $t$, whereas $h_{J_{t}}^{-}\left(M^{4}\right)$ is an upper-semi-continuous function in $t$, that is,

$$
h_{J_{t_{0}}}^{+}\left(M^{4}\right) \leq h_{J_{t}}^{+}\left(M^{4}\right), \quad h_{J_{t_{0}}}^{-}\left(M^{4}\right) \geq h_{J_{t}}^{-}\left(M^{4}\right),
$$

for every $t$ sufficiently close to $t_{0}$. Angella and Tomassini prove in [6] that these properties are no longer true in dimension $\geq 6$ by means of two explicit families of curves $J_{t}$. However, they show that their families $J_{t}$ are not $\mathcal{C}^{\infty}$-pure for $t \neq 0$ and pose the question of finding "more fulfilling counterexamples".

In [6, Proposition 4.3] a curve of almost-complex structures $J_{t}$ on $S^{3} \times \mathbb{T}^{3}$ is constructed in such a way that $J_{0}$ is a $\mathcal{C}^{\infty}$-full complex structure, $J_{t}$ is an almostcomplex structure which is not $\mathcal{C}^{\infty}$-pure for all $t$, and $h_{J_{0}}^{+}\left(S^{3} \times \mathbb{T}^{3}\right)=3>1=$ $h_{J_{t}}^{+}\left(S^{3} \times \mathbb{T}^{3}\right)$ for $t \neq 0$. In the following result we provide a curve of complex structures $J_{t}$ on $\mathcal{N}_{0}$, the nilmanifold underlying the Iwasawa manifold, which is constructed as an appropriate holomorphic deformation of the $\mathcal{C}^{\infty}$-pure-and-full Abelian structure $\mathcal{I}_{0}^{0}$ found in Theorem 2.3. This curve $J_{t}$ is $\mathcal{C}^{\infty}$-pure for all $t$ and still satisfies $h_{J_{0}}^{+}\left(\mathcal{N}_{0}\right)>h_{J_{t}}^{+}\left(\mathcal{N}_{0}\right)$ for $t \neq 0$.

Proposition 3.1. There exists a holomorphic family of compact complex manifolds $\left\{X_{t}=\left(M, J_{t}\right)\right\}_{t \in \mathbf{B}}$ of complex dimension 3 , where $\mathbf{B}=\{t \in \mathbb{C}|| t \mid<1\}$, such that:

(i) $X_{t}$ is $\mathcal{C}^{\infty}$-pure for every $t \in \mathbf{B}$;

(ii) $X_{0}$ is $\mathcal{C}^{\infty}$-full, but $X_{t}$ is not $\mathcal{C}^{\infty}$-full for $t \in \mathbf{B}-\{0\}$;

(iii) $h^{+}\left(X_{0}\right)>h^{+}\left(X_{t}\right)$ for any $t \in \mathbf{B}-\{0\}$.

Proof. The proof is based on an appropriate deformation of the $\mathcal{C}^{\infty}$-pure-and-full complex nilmanifold $\left(\mathcal{N}_{0}, \mathcal{I}_{0}^{0}\right)$ found in Theorem [2.3. Nevertheless, we will next consider the more general situation $\left(\mathcal{N}_{\epsilon}, \mathcal{I}_{\epsilon}^{0}\right)$, where $\epsilon=0$ or 1 ; that is, the complex structure equations are

$$
\mathcal{I}_{\epsilon}^{0}: \quad d \omega^{1}=0, \quad d \omega^{2}=\epsilon \omega^{1 \overline{1}}, \quad d \omega^{3}=\omega^{1 \overline{2}} .
$$

It is clear that the $(0,1)$-form $\omega^{\overline{3}}$ defines a Dolbeault cohomology class on $\left(\mathcal{N}_{\epsilon}, \mathcal{I}_{\epsilon}^{0}\right)$. Hence, we can choose the class $\left[\omega^{\overline{3}}\right] \in H_{\bar{\partial}}^{0,1}\left(\mathcal{N}_{\epsilon}, \mathcal{I}_{\epsilon}^{0}\right)$ to perform an appropriate holomorphic deformation of $\left(\mathcal{N}_{\epsilon}, \mathcal{I}_{\epsilon}^{0}\right)$. For each $t \in \mathbb{C}$ such that $|t|<1$, define the 
complex structure $J_{\epsilon}^{t}$ on $\mathcal{N}_{\epsilon}$ given by the following basis $\left\{\eta_{t}^{k}\right\}_{k=1}^{3}$ of $(1,0)$-forms:

$$
\eta_{t}^{1}:=\omega^{1}, \quad \eta_{t}^{2}:=\omega^{2}, \quad \eta_{t}^{3}:=\omega^{3}+t \omega^{\overline{3}} .
$$

The complex structure equations for $\left(\mathcal{N}_{\epsilon}, J_{\epsilon}^{t}\right)$ are

$$
d \eta_{t}^{1}=0, \quad d \eta_{t}^{2}=\epsilon \eta_{t}^{1 \overline{1}}, \quad d \eta_{t}^{3}=\eta_{t}^{1 \overline{2}}-t \eta_{t}^{2 \overline{1}} .
$$

Observe that the initial structure $\mathcal{I}_{\epsilon}^{0}$ is recovered for $t=0$. Since the complex structures $J_{\epsilon}^{t}$ are Abelian, they are $\mathcal{C}^{\infty}$-pure by Lemma 2.1.

A direct computation using (11) shows that for $\left(\mathcal{N}_{\epsilon}, J_{\epsilon}^{t}\right)$ one has

$$
\begin{aligned}
& H_{J_{\epsilon}^{t}}^{+}\left(\mathcal{N}_{\epsilon}\right)=\left\{\begin{array}{l}
\left\langle\left[i \eta_{t}^{1 \overline{1}}\right],\left[i \eta_{t}^{2 \overline{2}}\right], \delta_{t}\left[\eta_{t}^{2 \overline{3}}-\eta_{t}^{3 \overline{2}}\right], \delta_{t}\left[i\left(\eta_{t}^{2 \overline{3}}+\eta_{t}^{3 \overline{2}}\right)\right]\right\rangle, \text { if } \epsilon=0, \\
\left\langle\left[i\left(\eta_{t}^{1 \overline{3}}+\eta_{t}^{2 \overline{2}}+\eta_{t}^{3 \overline{1}}\right)\right]\right\rangle, \text { if } \epsilon=1,
\end{array}\right. \\
& H_{J_{\epsilon}^{t}}^{-}\left(\mathcal{N}_{\epsilon}\right)=\left\langle\left[\eta_{t}^{12}+\eta_{t}^{\overline{1} \overline{2}}\right],\left[i\left(\eta_{t}^{12}-\eta_{t}^{\overline{1} \overline{2}}\right)\right], \delta_{t}\left[\eta_{t}^{13}+\eta_{t}^{\overline{1} \overline{3}}\right], \delta_{t}\left[i\left(\eta_{t}^{13}-\eta_{t}^{\overline{1} \overline{3}}\right)\right]\right\rangle,
\end{aligned}
$$

together with the following relation for de Rham cohomology classes:

$$
\left[\eta_{t}^{1 \overline{2}}-\eta_{t}^{2 \overline{1}}\right]=\left[i\left(\eta_{t}^{1 \overline{2}}+\eta_{t}^{2 \overline{1}}\right)\right]=\mathbf{0},
$$

and the additional condition $\left[i \eta_{t}^{1 \overline{1}}\right]=\mathbf{0}$ only for the case $\epsilon=1$.

The previous description allows us to conclude that, for any $t \neq 0$, the complex structure $J_{\epsilon}^{t}$ is $\mathcal{C}^{\infty}$-pure but not $\mathcal{C}^{\infty}$-full.

Moreover, when $\epsilon=0$, one has $h_{\mathcal{I}_{0}^{0}}^{+}\left(\mathcal{N}_{0}\right)=4>2=h_{J_{0}^{t}}^{+}\left(\mathcal{N}_{0}\right)$ for any $t \in \mathbf{B}-\{0\}$. Notice that $h_{\mathcal{I}_{0}^{0}}^{-}\left(\mathcal{N}_{0}\right)=4>2=h_{J_{0}^{t}}^{-}\left(\mathcal{N}_{0}\right)$ for $t \neq 0$.

Remark 3.2. It follows from the previous proof that there is a holomorphic deformation $X_{t}$ of the $\mathcal{C}^{\infty}$-pure-and-full complex nilmanifold $\left(\mathcal{N}_{1}, \mathcal{I}_{1}^{0}\right)$ satisfying the properties (i) and (ii) in Proposition 3.1. Nonetheless, $h^{+}\left(X_{t}\right)$ remains constant along this deformation.

In [6, Proposition 4.1] one can find a curve of complex structures $J_{t}$ on a 10 dimensional nilmanifold such that $J_{0}$ is $\mathcal{C}^{\infty}$-pure-and-full, $J_{t}$ is not $\mathcal{C}^{\infty}$-pure for $t \neq 0$ and $h_{J_{0}}^{-}=10<12=h_{J_{t}}^{-}$for $t \neq 0$. In the following result we construct a deformation in dimension 6 with a similar behaviour but also satisfying the $\mathcal{C}^{\infty}$-full property at every fiber of it. The proof is based on an appropriate holomorphic deformation of the $\mathcal{C}^{\infty}$-pure-and-full complex nilmanifold $\left(\mathcal{N}_{1}, \mathcal{I}_{1}^{1}\right)$ found in Theorem 2.3

Proposition 3.3. There exists a holomorphic family of compact complex manifolds $\left\{X_{t}=\left(M, J_{t}\right)\right\}_{t \in \mathbf{B}}$ of complex dimension 3 , where $\mathbf{B}=\{t \in \mathbb{C}|| t \mid<1\}$, such that:

(i) $X_{t}$ is $\mathcal{C}^{\infty}$-full for every $t \in \mathbf{B}$;

(ii) $X_{0}$ is $\mathcal{C}^{\infty}$-pure, but $X_{t}$ is not $\mathcal{C}^{\infty}$-pure for $t \in \mathbf{B}-\{0\}$;

(iii) $h^{-}\left(X_{0}\right)<h^{-}\left(X_{t}\right)$ for any $t \in \mathbf{B}-\{0\}$.

Proof. Let us take $J_{0}$ as the $\mathcal{C}^{\infty}$-pure-and-full complex structure $\mathcal{I}_{1}^{1}$ on the nilmanifold $\mathcal{N}_{1}$ found in Theorem 2.3. Now, as the form $\omega^{\overline{2}}$ defines a non-zero Dolbeault cohomology class in $H_{\bar{\partial}}^{0,1}\left(\mathcal{N}_{1}, \mathcal{I}_{1}^{1}\right)$, it will be used to perform the following holomorphic deformation of $\left(\mathcal{N}_{1}, \mathcal{I}_{1}^{1}\right)$. For each $t \in \mathbb{C}$ such that $|t|<1$, we define the complex structure $J_{t}$ on $\mathcal{N}_{1}$ by the following basis $\left\{\eta_{t}^{k}\right\}_{k=1}^{3}$ of $(1,0)$-forms:

$$
\eta_{t}^{1}:=\omega^{1}, \quad \eta_{t}^{2}:=\omega^{2}+t \omega^{\overline{2}}, \quad \eta_{t}^{3}:=\omega^{3} .
$$


The complex structure equations for $\left(\mathcal{N}_{1}, J_{t}\right)$ are

$$
d \eta_{t}^{1}=0, \quad d \eta_{t}^{2}=(1-t) \eta_{t}^{1 \overline{1}}, \quad d \eta_{t}^{3}=\frac{1}{1-|t|^{2}} \eta_{t}^{12}-\frac{t}{1-|t|^{2}} \eta_{t}^{1 \overline{2}} .
$$

With respect to the new $(1,0)$-basis for $J_{t}$, given by

$$
\tau_{t}^{1}=\eta_{t}^{1}, \quad \tau_{t}^{2}=\frac{1}{1-t} \eta_{t}^{2}, \quad \tau_{t}^{3}=\frac{1-|t|^{2}}{1-t} \eta_{t}^{3},
$$

we can rewrite the previous structure equations in a simpler way as

$$
d \tau_{t}^{1}=0, \quad d \tau_{t}^{2}=\tau_{t}^{1 \overline{1}}, \quad d \tau_{t}^{3}=\tau_{t}^{12}+B \tau_{t}^{1 \overline{2}},
$$

where $B=-\frac{t(1-\bar{t})}{1-t}$. Notice that the initial complex structure $\mathcal{I}_{1}^{1}$ is recovered for $t=0$. A direct calculation using (2) leads to

$$
\begin{aligned}
& H_{J_{t}}^{+}\left(\mathcal{N}_{1}\right)=\left\langle\left[\tau_{t}^{1 \overline{2}}-\tau_{t}^{2 \overline{1}}\right],\left[i\left(\tau_{t}^{1 \overline{2}}+\tau_{t}^{2 \overline{1}}\right)\right],\left[i\left((B-1) \tau_{t}^{1 \overline{3}}+\left(|B|^{2}-1\right) \tau_{t}^{2 \overline{2}}+(\bar{B}-1) \tau_{t}^{3 \overline{1}}\right)\right]\right\rangle, \\
& H_{J_{t}}^{-}\left(\mathcal{N}_{1}\right)=\left\langle\left[\tau_{t}^{13}+\tau_{t}^{\overline{1} \overline{3}}\right],\left[i\left(\tau_{t}^{13}-\tau_{t}^{\overline{1} \overline{3}}\right)\right],\left(1-\delta_{B}\right)\left[\tau_{t}^{12}+\tau_{t}^{\overline{1} \overline{2}}\right],\left(1-\delta_{B}\right)\left[i\left(\tau_{t}^{12}-\tau_{t}^{\overline{1} \overline{2}}\right)\right]\right\rangle .
\end{aligned}
$$

Furthermore, the following cohomological relations hold:

$$
\begin{aligned}
{\left[\tau_{t}^{12}+\tau_{t}^{\overline{1} \overline{2}}\right] } & =-\mathfrak{R e} B\left[\left(\tau_{t}^{1 \overline{2}}-\tau_{t}^{2 \overline{1}}\right)\right]-\mathfrak{I m} B\left[i\left(\tau_{t}^{1 \overline{2}}+\tau_{t}^{2 \overline{1}}\right)\right], \\
{\left[i\left(\tau_{t}^{12}-\tau_{t}^{\overline{1} \overline{2}}\right)\right] } & =\mathfrak{I m} B\left[\left(\tau_{t}^{1 \overline{2}}-\tau_{t}^{2 \overline{1}}\right)\right]-\mathfrak{R e} B\left[i\left(\tau_{t}^{1 \overline{2}}+\tau_{t}^{2 \overline{1}}\right)\right] .
\end{aligned}
$$

Observe that when $t=0$ (i.e. $B=0$ ), one has that $\left[\tau_{t}^{12}+\tau_{t}^{\overline{1} \overline{2}}\right]=\left[i\left(\tau_{t}^{12}-\tau_{t}^{\overline{1} \overline{2}}\right)\right]=\mathbf{0}$. Otherwise, one can conclude that $J_{t}$ with $t \neq 0$ (i.e. $B \neq 0$ ) is not $\mathcal{C}^{\infty}$-pure, although it is $\mathcal{C}^{\infty}$-full because the second Betti number of the nilmanifold $\mathcal{N}_{1}$ equals 5 .

Finally, counting dimensions we arrive at $h_{J_{0}}^{-}\left(\mathcal{N}_{1}\right)=2<4=h_{J_{t}}^{-}\left(\mathcal{N}_{1}\right)$ for any $t \neq 0$. Notice that $h_{J_{t}}^{+}\left(\mathcal{N}_{1}\right)=3$ remains constant.

3.1. Small deformations of the Iwasawa manifold revisited. In this section we focus on the small deformations of the Iwasawa manifold $\left(\mathcal{N}_{0}, \mathcal{I}_{0}^{1}\right)$, following the approach in [5] (see also [1,2]). Let us recall that Nakamura studied in [21] the small deformations of the Iwasawa manifold, dividing them into three different classes according to their Hodge diamond. Each class was characterized as follows:

Class (i): $t_{11}=t_{12}=t_{21}=t_{22}=0$,

Class (ii): $\mathcal{D}(\mathbf{t})=0$ and $\left(t_{11}, t_{12}, t_{21}, t_{22}\right) \neq(0,0,0,0)$,

Class (iii): $\mathcal{D}(\mathbf{t}) \neq 0$,

where $t_{11}, t_{12}, t_{21}, t_{22}$ are parameters in the deformation space

$$
\mathbf{B}=\left\{\mathbf{t}=\left(t_{11}, t_{12}, t_{21}, t_{22}, t_{31}, t_{32}\right) \in \mathbb{C}^{6}|| \mathbf{t} \mid<\varepsilon\right\}
$$

for a sufficiently small $\varepsilon>0$, and $\mathcal{D}(\mathbf{t})=t_{11} t_{22}-t_{12} t_{21}$.

In [5], Angella and Tomassini proved that only class $(i)$ is $\mathcal{C}^{\infty}$-pure-and-full, and, as a consequence, they conclude that $h^{+}=h^{-}=4$ for both the Iwasawa manifold and any small deformation in the class (i). The remaining classes (ii) and (iii) are proved to be neither $\mathcal{C}^{\infty}$-pure nor $\mathcal{C}^{\infty}$-full, although the dimensions $h^{+}$and $h^{-}$are not determined. The following result shows that $h^{+}$remains constant and equal to 4 for any small deformation of the Iwasawa manifold.

Proposition 3.4. For any sufficiently small deformation $X_{\mathbf{t}}$ of the Iwasawa manifold one has $h^{+}\left(X_{\mathbf{t}}\right)=4$. 
Proof. The result is known for $X_{\mathbf{t}}$ in the class $(i)$. For the classes $(i i)$ and $(i i i)$, one can proceed as in the proof of [5. Theorem 3.1] and write the complex structure equations of $X_{\mathbf{t}}$ as

$$
\left\{\begin{array}{l}
d \varphi_{\mathbf{t}}^{1}=d \varphi_{\mathbf{t}}^{2}=0 \\
d \varphi_{\mathbf{t}}^{3}=\sigma_{12} \varphi_{\mathbf{t}}^{12}+\sigma_{1 \overline{1}} \varphi_{\mathbf{t}}^{1 \overline{1}}+\sigma_{1 \overline{2}} \varphi_{\mathbf{t}}^{1 \overline{2}}+\sigma_{2 \overline{1}} \varphi_{\mathbf{t}}^{2 \overline{1}}+\sigma_{2 \overline{2}} \varphi_{\mathbf{t}}^{2 \overline{2}},
\end{array}\right.
$$

where the coefficients $\sigma_{12}, \sigma_{1 \overline{1}}, \sigma_{1 \overline{2}}, \sigma_{2 \overline{1}}, \sigma_{2 \overline{2}} \in \mathbb{C}$ depend only on $\mathbf{t}$ and are given by

$$
\begin{aligned}
\sigma_{12} & =-\gamma-\bar{\alpha}\left|t_{22}\right|^{2}+\frac{1}{\bar{\gamma}}\left(\sigma_{1 \overline{1}} \bar{\sigma}_{2 \overline{2}}\right), \\
\sigma_{1 \overline{1}} & =\bar{\alpha} \bar{\gamma}\left(t_{21}+\bar{t}_{21} \mathcal{D}(\mathbf{t})\right), \\
\sigma_{1 \overline{2}} & =\bar{\alpha}\left(t_{22}+\left(t_{12} \bar{t}_{11}+t_{22} \bar{t}_{12}\right) \sigma_{1 \overline{1}}\right), \\
\sigma_{2 \overline{1}} & =-\alpha \gamma\left(t_{11}-\bar{t}_{22} \mathcal{D}(\mathbf{t})\right), \\
\sigma_{2 \overline{2}} & =-\alpha \gamma\left(t_{12}+\bar{t}_{12} \mathcal{D}(\mathbf{t})\right),
\end{aligned}
$$

with $\alpha$ and $\gamma$ satisfying

$$
\begin{aligned}
\alpha & =\frac{1}{1-\left|t_{22}\right|^{2}-t_{21} \bar{t}_{12}}, \\
\gamma & =\frac{1}{1-\left|t_{11}\right|^{2}-t_{12} \bar{t}_{21}-\alpha\left(\left|t_{11}\right|^{2} t_{21} \bar{t}_{12}+\left|t_{22}\right|^{2} t_{12} \bar{t}_{21}+2 \mathfrak{R e}\left(t_{11} t_{22} \bar{t}_{12} \bar{t}_{21}\right)\right)} .
\end{aligned}
$$

Observe that some of the coefficients have been rewritten in a different way with respect to [5], which will be more suitable for our purpose.

In order to compute the dimension of $H^{+}\left(X_{\mathbf{t}}\right)$, we first determine the space of invariant closed $(1,1)$-forms on $X_{\mathbf{t}}$. It is clear that the $(1,1)$-forms $\varphi_{\mathbf{t}}^{1 \overline{1}}, \varphi_{\mathbf{t}}^{1 \overline{2}}, \varphi_{\mathbf{t}}^{2 \overline{1}}$ and $\varphi_{\mathbf{t}}^{2 \overline{2}}$ are closed. To see whether there exist any other closed $(1,1)$-forms, consider

$$
\Phi=a \varphi_{\mathbf{t}}^{1 \overline{3}}+b \varphi_{\mathbf{t}}^{2 \overline{3}}+c \varphi_{\mathbf{t}}^{3 \overline{1}}+e \varphi_{\mathbf{t}}^{3 \overline{2}}+f \varphi_{\mathbf{t}}^{3 \overline{3}}
$$

where $a, b, c, e, f \in \mathbb{C}$. The condition $d \Phi=0$ leads to $f=0$ and to the system of equations:

$$
\left\{\begin{array}{l}
a \bar{\sigma}_{1 \overline{2}}-b \bar{\sigma}_{1 \overline{1}}+c \sigma_{12}=0, \\
a \bar{\sigma}_{2 \overline{2}}-b \bar{\sigma}_{2 \overline{1}}+e \sigma_{12}=0, \\
a \bar{\sigma}_{12}+c \sigma_{1 \overline{2}}-e \sigma_{1 \overline{1}}=0 \\
b \bar{\sigma}_{12}+c \sigma_{2 \overline{2}}-e \sigma_{2 \overline{1}}=0 .
\end{array}\right.
$$

Due to the fact that $\sigma_{12}$ is non-zero for any sufficiently small $\mathbf{t}$, one has

$$
c=\frac{b \bar{\sigma}_{1 \overline{1}}-a \bar{\sigma}_{1 \overline{2}}}{\sigma_{12}} \text { and } e=\frac{b \bar{\sigma}_{2 \overline{1}}-a \bar{\sigma}_{2 \overline{2}}}{\sigma_{12}},
$$

but also

$$
\left(\begin{array}{cc}
\left|\sigma_{12}\right|^{2}-\left|\sigma_{1 \overline{2}}\right|^{2}+\sigma_{1 \overline{1}} \bar{\sigma}_{2 \overline{2}} & \sigma_{1 \overline{2}} \bar{\sigma}_{1 \overline{1}}-\sigma_{1 \overline{1}} \bar{\sigma}_{2 \overline{1}} \\
\sigma_{2 \overline{1}} \bar{\sigma}_{2 \overline{2}}-\sigma_{2 \overline{2}} \bar{\sigma}_{1 \overline{2}} & \left|\sigma_{12}\right|^{2}-\left|\sigma_{2 \overline{1}}\right|^{2}+\sigma_{2 \overline{2}} \bar{\sigma}_{1 \overline{1}}
\end{array}\right)\left(\begin{array}{l}
a \\
b
\end{array}\right)=\left(\begin{array}{l}
0 \\
0
\end{array}\right) .
$$

Since the determinant of the previous matrix never vanishes for any sufficiently small $\mathbf{t}$, we conclude that $a=b=0$ and thus $\Phi=0$. Therefore, the space of invariant closed $(1,1)$-forms on $X_{\mathbf{t}}$ is generated by $\varphi_{\mathbf{t}}^{i \bar{k}}$, for $i, k=1,2$. Furthermore, any such form is never $d$-exact because the coefficient $\sigma_{12}$ does not vanish for sufficiently small $\mathbf{t}$. Then, we have

$$
H^{+}\left(X_{\mathbf{t}}\right)=\left\langle\left[i \varphi_{\mathbf{t}}^{1 \overline{1}}\right],\left[i \varphi_{\mathbf{t}}^{2 \overline{2}}\right],\left[\varphi_{\mathbf{t}}^{1 \overline{2}}-\varphi_{\mathbf{t}}^{2 \overline{1}}\right],\left[i\left(\varphi_{\mathbf{t}}^{1 \overline{2}}+\varphi_{\mathbf{t}}^{2 \overline{1}}\right)\right]\right\rangle,
$$


for any sufficiently small deformation $X_{\mathbf{t}}$ in the classes (ii) and (iii). This concludes the proof.

The following example shows how drastically the subspace $H^{-}$may change along a deformation.

Example 3.5. There exists a small deformation $X_{\mathbf{t}}$ of the Iwasawa manifold satisfying $h^{-}\left(X_{\mathbf{t}}\right)=1$ and $H^{-}\left(X_{\mathbf{t}}\right) \subset H^{+}\left(X_{\mathbf{t}}\right)$ for every $\mathbf{t} \neq 0$. It can be directly seen from (3) and (4) that $h^{-}\left(X_{\mathbf{t}}\right) \geq 1$ for any small deformation $X_{\mathbf{t}}$ of the Iwasawa manifold. We next show a particular deformation with $h^{-}\left(X_{\mathbf{t}}\right)=1$ such that $H^{-}\left(X_{\mathbf{t}}\right) \subset H^{+}\left(X_{\mathbf{t}}\right)$. Let us consider the small deformation given by $t_{12}=t_{21}=0$ and $t_{11}=t_{22}=t$, with $|t|<\varepsilon$. Observe that it belongs to class (iii). The structure equations are given by (3), and to find their coefficients, it suffices to replace our specific values of $t_{11}, t_{12}, t_{21}$ and $t_{22}$ in (4):

$$
\sigma_{12}=-\frac{1+|t|^{2}}{1-|t|^{2}}, \quad \sigma_{1 \overline{1}}=0, \quad \sigma_{1 \overline{2}}=\frac{t}{1-|t|^{2}}, \quad \sigma_{2 \overline{1}}=-\frac{t}{1-|t|^{2}}, \quad \text { and } \quad \sigma_{2 \overline{2}}=0 ;
$$

that is, the complex structure equations of $X_{\mathbf{t}}$ are

$$
d \varphi_{\mathbf{t}}^{1}=d \varphi_{\mathbf{t}}^{2}=0, \quad d \varphi_{\mathbf{t}}^{3}=-\frac{1+|t|^{2}}{1-|t|^{2}} \varphi_{\mathbf{t}}^{12}+\frac{t}{1-|t|^{2}} \varphi_{\mathbf{t}}^{1 \overline{2}}-\frac{t}{1-|t|^{2}} \varphi_{\mathbf{t}}^{2 \overline{1}} .
$$

For $t \neq 0$, a direct computation shows that only $\varphi^{12}+\varphi^{\overline{1} \overline{2}}$ and $i\left(\varphi^{12}-\varphi^{\overline{1} \overline{2}}\right)$ define a cohomology class in $H^{-}\left(X_{\mathbf{t}}\right)$. However, the equality

$$
(\mathfrak{I m} t)\left(\varphi_{\mathbf{t}}^{12}+\varphi_{\mathbf{t}}^{\overline{1} \overline{2}}\right)+(\mathfrak{R e} t) i\left(\varphi_{\mathbf{t}}^{12}-\varphi_{\mathbf{t}}^{\overline{1} \overline{2}}\right)=-\frac{1-|t|^{2}}{1+|t|^{2}} d\left(i \bar{t} \varphi_{\mathbf{t}}^{3}-i t \varphi_{\mathbf{t}}^{\overline{3}}\right)
$$

implies that $h^{-}\left(X_{\mathbf{t}}\right)=1$. Moreover, the space $H^{-}\left(X_{\mathbf{t}}\right)$ is contained in $H^{+}\left(X_{\mathbf{t}}\right)$ for $t \neq 0$ because the following relations are satisfied:

$$
\begin{aligned}
{\left[\varphi_{\mathbf{t}}^{12}+\varphi_{\mathbf{t}}^{\overline{1} \overline{2}}\right] } & =\frac{2 \mathfrak{R e} t}{1+|t|^{2}}\left[\varphi_{\mathbf{t}}^{1 \overline{2}}-\varphi_{\mathbf{t}}^{2 \overline{1}}\right] \in H^{+}\left(X_{\mathbf{t}}\right), \\
{\left[i\left(\varphi_{\mathbf{t}}^{12}-\varphi_{\mathbf{t}}^{\overline{1} \overline{2}}\right)\right] } & =-\frac{2 \mathfrak{I m} t}{1+|t|^{2}}\left[\varphi_{\mathbf{t}}^{1 \overline{2}}-\varphi_{\mathbf{t}}^{2 \overline{1}}\right] \in H^{+}\left(X_{\mathbf{t}}\right) .
\end{aligned}
$$

\section{The $\mathcal{C}^{\infty}$-PURE-AND-FULL PROPERTY IS NOT CLOSED UNDER HOLOMORPHIC DEFORMATIONS}

Following [23, Definition 1.12], let $\mathbf{B}$ be an open disc around the origin in $\mathbb{C}$. A property is said to be closed under holomorphic deformations if, for every holomorphic family of compact complex manifolds $\left(X_{t}\right)_{t \in \mathbf{B}}$, whenever $X_{t}$ has the property for all $t \in \mathbf{B}-\{0\}$, then the property also holds in the central limit $X_{0}$. Our goal in this section is to prove that the $\mathcal{C}^{\infty}$-pure-and-full property is not closed under holomorphic deformations.

We will use the results about 6-dimensional solvmanifolds with holomorphically trivial canonical bundle obtained in [15. Concretely, let $G$ be the 6-dimensional simply-connected solvable Lie group whose Lie algebra $\mathfrak{g}$ is defined by the following structure equations:

$$
\left\{\begin{aligned}
d e^{1}=e^{16}-e^{25}, & d e^{3}=-e^{36}+e^{45}, & d e^{5}=0, \\
d e^{2}=e^{15}+e^{26}, & d e^{4}=-e^{35}-e^{46}, & d e^{6}=0 .
\end{aligned}\right.
$$


Let us consider the left-invariant almost complex structure $J$ on the Lie group $G$ given by

$$
J e^{1}=e^{2}-e^{5}, \quad J e^{2}=-e^{1}-e^{6}, \quad J e^{3}=e^{4}, \quad J e^{4}=-e^{3}, \quad J e^{5}=-e^{6}, \quad J e^{6}=e^{5} .
$$

The forms

$$
\omega^{1}=\frac{1}{2}\left(e^{1}-i\left(e^{2}-e^{5}\right)\right), \quad \omega^{2}=e^{3}-i e^{4}, \quad \omega^{3}=-\frac{1}{2}\left(e^{5}+i e^{6}\right)
$$

have bidegree $(1,0)$ with respect to $J$, and they satisfy

$$
d \omega^{1}=2 i \omega^{13}+\omega^{3 \overline{3}}, \quad d \omega^{2}=-2 i \omega^{23}, \quad d \omega^{3}=0 .
$$

Hence, $J$ is integrable and it defines a left-invariant complex structure on $G$.

The Lie algebra $\mathfrak{g}$ is precisely the real Lie algebra underlying the Nakamura manifold, and the Lie group $G$ admits lattices $\Gamma$ [4,21]. Hence, $J$ defines a complex structure on any compact quotient solvmanifold $\Gamma \backslash G$.

Based on a result in [15, in the following theorem we show that there is a lattice and a holomorphic deformation of the complex structure $J$ showing that being $\mathcal{C}^{\infty}$-pure-and-full is not a closed property.

Theorem 4.1. There exists a holomorphic family of compact complex manifolds $\left(X_{t}\right)_{t \in \mathbf{B}}$, where $\mathbf{B}=\{t \in \mathbb{C}|| t \mid<1\}$, such that $X_{t}$ is $\mathcal{C}^{\infty}$-pure-and-full for every $t \in \mathbf{B}-\{0\}$, but $X_{0}$ is neither $\mathcal{C}^{\infty}$-pure nor $\mathcal{C}^{\infty}$-full.

Proof. Let $\Gamma \backslash G$ be any compact solvmanifold endowed with the complex structure $J$ defined above. By the equations (5), the conjugate of the $(1,0)$-form $\omega^{3}$ defines a Dolbeault cohomology class, i.e. $\left[\omega^{\overline{3}}\right] \in H_{\bar{\partial}}^{0,1}(\Gamma \backslash G, J)$. Hence, for any $t \in \mathbf{B}=$ $\{t \in \mathbb{C}|| t \mid<1\}$, we define the complex structure $J_{t}$ on $\Gamma \backslash G$ given by the following basis $\left\{\eta_{t}^{k}\right\}_{k=1}^{3}$ of $(1,0)$-forms:

$$
\eta_{t}^{1}:=\omega^{1}, \quad \eta_{t}^{2}:=\omega^{2}, \quad \eta_{t}^{3}:=\omega^{3}+t \omega^{\overline{3}} .
$$

The complex structure equations for $\left(\Gamma \backslash G, J_{t}\right)$ are

$$
\left\{\begin{array}{l}
d \eta_{t}^{1}=\frac{2 i}{1-|t|^{2}} \eta_{t}^{13}-\frac{2 i t}{1-|t|^{2}} \eta_{t}^{1 \overline{3}}+\frac{1}{1-|t|^{2}} \eta_{t}^{3 \overline{3}} \\
d \eta_{t}^{2}=-\frac{2 i}{1-|t|^{2}} \eta_{t}^{23}+\frac{2 i t}{1-|t|^{2}} \eta_{t}^{2 \overline{3}} \\
d \eta_{t}^{3}=0
\end{array}\right.
$$

In [15, Theorem 5.2] it is shown that every complex structure $J_{t}$ with $t \neq 0$ is equivalent to a complex structure obtained in [4] as a certain deformation of the Nakamura manifold. In [4, Proposition 4.1] it is proved that there is a lattice $\Gamma$ in $G$ such that the corresponding solvmanifold $M=\Gamma \backslash G$ endowed with the complex structure $J_{t}$ satisfies the $\partial \bar{\partial}$-Lemma for any $t \neq 0$. Let us consider the holomorphic family of compact complex manifolds $X_{t}=\left(M, J_{t}\right), t \in \mathbf{B}$. For every $t \in \mathbf{B}-\{0\}$, it is clear that the manifold $X_{t}$ is $\mathcal{C}^{\infty}$-pure-and-full, as any compact complex manifold satisfying the $\partial \bar{\partial}$-Lemma is.

Next we see that the central limit $X_{0}=\left(M, J_{0}\right)$ of the holomorphic family $\left(X_{t}\right)_{t \in \mathbf{B}}$ is neither $\mathcal{C}^{\infty}$-pure nor $\mathcal{C}^{\infty}$-full. Since $\eta_{0}^{k}=\omega^{k}$, the complex structure equations (6) for $t=0$ are precisely (5). The (real) form $i \omega^{3 \overline{3}}$ is a closed $J_{0}$-invariant 2 -form, and the form $\omega^{13}+\omega^{\overline{1} \overline{3}}$ is a real closed 2 -form which is $J_{0}$-anti-invariant. Therefore, they define cohomology classes

$$
\left[i \omega^{3 \overline{3}}\right] \in H_{J_{0}}^{+}(M), \quad\left[\omega^{13}+\omega^{\overline{1} \overline{3}}\right] \in H_{J_{0}}^{-}(M) .
$$


Moreover, these classes are non-zero in $H_{\mathrm{dR}}^{2}(M ; \mathbb{R})$. To see this, we can apply the well-known symmetrization process, which consists of the map $\sim: \Omega^{k}(M) \longrightarrow$ $\bigwedge^{k}\left(\mathfrak{g}^{*}\right)$ sending any form $\alpha \in \Omega^{k}(M)$ to an element $\tilde{\alpha} \in \bigwedge^{k}\left(\mathfrak{g}^{*}\right)$ obtained by integration on the solvmanifold $M=\Gamma \backslash G$ with respect to a volume element induced by a bi-invariant one on the Lie group $G$. We will next use that symmetrization commutes with $d$ and, since the complex structure $J_{0}$ is invariant, it also preserves the bigraduation induced by $J_{0}$ on the complex forms. Thus, since the invariant forms $i \omega^{3 \overline{3}}$ and $\omega^{13}+\omega^{\overline{1} \overline{3}}$ do not belong to the space $d\left(\bigwedge^{1}\left(\mathfrak{g}^{*}\right)\right)$, we conclude that their cohomology classes in (7) are non-zero.

However, from (5) we see that

$$
i \omega^{3 \overline{3}}-\left(\omega^{13}+\omega^{\overline{1} \overline{3}}\right)=\frac{1}{2} d\left(i \omega^{1}-i \omega^{\overline{1}}\right) .
$$

Hence,

$$
\mathbf{0} \neq\left[i \omega^{3 \overline{3}}\right]=\left[\omega^{13}+\omega^{\overline{1} \overline{3}}\right] \in H_{J_{0}}^{+}(M) \cap H_{J_{0}}^{-}(M),
$$

and $X_{0}$ is not $\mathcal{C}^{\infty}$-pure.

Now we prove that $X_{0}$ is not $\mathcal{C}^{\infty}$-full. From the equations (5) we have that the real 2-form $2 i \omega^{12}+\omega^{2 \overline{3}}-\omega^{3 \overline{2}}-2 i \omega^{\overline{1} \overline{2}}$ is closed and it defines a de Rham cohomology class

$$
\left[2 i \omega^{12}+\omega^{2 \overline{3}}-\omega^{3 \overline{2}}-2 i \omega^{\overline{1} \overline{2}}\right] \in H_{\mathrm{dR}}^{2}(M ; \mathbb{R}) .
$$

Suppose that the equality $H_{\mathrm{dR}}^{2}(M ; \mathbb{R})=H_{J_{0}}^{+}(M)+H_{J_{0}}^{-}(M)$ holds. Then, there exist $\mathbf{a} \in H_{J_{0}}^{+}(M)$ and $\mathbf{b} \in H_{J_{0}}^{-}(M)$ such that $\left[2 i \omega^{12}+\omega^{2 \overline{3}}-\omega^{3 \overline{2}}-2 i \omega^{\overline{1} \overline{2}}\right]=\mathbf{a}+\mathbf{b}$. Equivalently, there are $\alpha \in \mathcal{Z}_{J_{0}}^{+}(M), \beta \in \mathcal{Z}_{J_{0}}^{-}(M)$, and $\gamma \in \Omega^{1}(M)$ such that

$$
2 i \omega^{12}+\omega^{2 \overline{3}}-\omega^{3 \overline{2}}-2 i \omega^{\overline{1} \overline{2}}=\alpha+\beta+d \gamma,
$$

where $\mathcal{Z}_{J_{0}}^{+}$, resp. $\mathcal{Z}_{J_{0}}^{-}$, is the space of real closed 2 -forms that are $J_{0}$-invariant, resp. $J_{0}$-anti-invariant.

Applying the symmetrization process to (8), we get

$$
2 i \omega^{12}+\omega^{2 \overline{3}}-\omega^{3 \overline{2}}-2 i \omega^{\overline{1} \overline{2}}=\tilde{\alpha}+\tilde{\beta}+d \tilde{\gamma},
$$

for some $\tilde{\alpha} \in \mathcal{Z}_{J_{0}}^{+}\left(\mathfrak{g}^{*}\right), \tilde{\beta} \in \mathcal{Z}_{J_{0}}^{-}\left(\mathfrak{g}^{*}\right)$, and $\tilde{\gamma} \in \bigwedge^{1}\left(\mathfrak{g}^{*}\right)$. However, this is not possible because a direct calculation from (5) yields

$\mathcal{Z}_{J_{0}}^{+}\left(\mathfrak{g}^{*}\right)+\mathcal{Z}_{J_{0}}^{-}\left(\mathfrak{g}^{*}\right)+d\left(\bigwedge^{1}\left(\mathfrak{g}^{*}\right)\right)=\left\langle i \omega^{3 \overline{3}}, \omega^{13}+\omega^{\overline{1} \overline{3}}, i\left(\omega^{13}-\omega^{\overline{1} \overline{3}}\right), \omega^{23}+\omega^{\overline{2} \overline{3}}, i\left(\omega^{23}-\omega^{\overline{2} \overline{3}}\right)\right\rangle$.

In conclusion, $\left[2 i \omega^{12}+\omega^{2 \overline{3}}-\omega^{3 \overline{2}}-2 i \omega^{\overline{1} \overline{2}}\right] \notin H_{J_{0}}^{+}(M)+H_{J_{0}}^{-}(M)$, and thus $X_{0}$ is not $\mathcal{C}^{\infty}$-full.

As a consequence of Theorem 4.1 we have:

Corollary 4.2. For compact complex manifolds, the following properties:

(i) "being $\mathcal{C}^{\infty}$-pure",

(ii) "being $\mathcal{C}^{\infty}$-full",

(iii) "being $\mathcal{C}^{\infty}$-pure-and-full",

are not closed under holomorphic deformations. 


\section{RELATiOn OF THE $\mathcal{C}^{\infty}$-PURE-AND-FULl PROPERTY WITH OTHER COMPLEX INVARIANTS AND METRIC PROPERTIES}

In this section we use the classification of complex nilmanifolds satisfying the $\mathcal{C}^{\infty}$-pure-and-full property obtained in Section 2 to illustrate that this property is unrelated to other metric properties. We will finish showing a possible relation to other complex invariants of the manifold.

Recall that Enrietti, Fino, and Vezzoni proved in [14] that an invariant complex structure $J$ on a nilmanifold $M$ is tamed by a symplectic form if and only if $(M, J)$ is a complex torus. Any Hermitian-symplectic structure is in particular SKT, i.e. the fundamental form $F$ satisfies $\partial \bar{\partial} F=0$; and if an SKT metric exists on $(M, J)$, then the nilmanifold is at most 2-step.

Another well-known and interesting class of Hermitian metrics is the one constituted by the locally conformal Kähler metrics, defined by the condition $d F=\theta \wedge F$, where $\theta$ is the (closed) Lee form. If $\theta$ is in addition a nowhere vanishing parallel form, then $F$ is called a Vaisman metric.

Two important classes of metrics defined by a condition on the $(2 n-2)$-form $F^{n-1}$, being $n$ the complex dimension of the manifold, are given by the balanced metrics [20] and the strongly Gauduchon metrics [22]. Recall that a Hermitian metric is called balanced if the fundamental form $F$ satisfies that $F^{n-1}$ is closed, and it is said to be strongly Gauduchon if $\partial F^{n-1}$ is $\bar{\partial}$-exact. Obviously, any balanced metric is strongly Gauduchon. Our first question in this section is whether the $\mathcal{C}^{\infty}$ pure-and-full property implies the existence of some special Hermitian metric on the manifold.

Proposition 5.1. The compact complex nilmanifolds $\left(\mathcal{N}_{1}, \mathcal{I}_{1}^{\rho}\right), \rho \in\{0,1\}$, satisfy the $\mathcal{C}^{\infty}$-pure-and-full property, but they do not admit any SKT, locally conformal Kähler, or strongly Gauduchon metrics.

Proof. By Theorem 2.3 we know that the complex structures $\mathcal{I}_{1}^{\rho}$ are $\mathcal{C}^{\infty}$-pure-andfull for $\rho=0,1$. The non-existence of SKT, locally conformal Kähler or strongly Gauduchon metrics can be derived from classification results in [9, 14, 26]. Nevertheless, for the sake of completeness we provide here a more direct and unified proof. First notice that by symmetrization one can reduce the problem to simply studying invariant Hermitian metrics. Consider the complex equations in Theorem 2.3 for $\epsilon=1$, and let $F$ be the fundamental 2-form of a generic invariant Hermitian metric, i.e.

$$
2 F=i\left(r^{2} \omega^{1 \overline{1}}+s^{2} \omega^{2 \overline{2}}+t^{2} \omega^{3 \overline{3}}\right)+u \omega^{1 \overline{2}}-\bar{u} \omega^{2 \overline{1}}+v \omega^{2 \overline{3}}-\bar{v} \omega^{3 \overline{2}}+z \omega^{1 \overline{3}}-\bar{z} \omega^{3 \overline{1}} .
$$

A direct calculation gives

(9) $2 \partial F=-\left(i s^{2}+\rho \bar{z}-(1-\rho) z\right) \omega^{12 \overline{1}}-\rho \bar{v} \omega^{12 \overline{2}}+i \rho t^{2} \omega^{12 \overline{3}}+\bar{v} \omega^{13 \overline{1}}-i(1-\rho) t^{2} \omega^{23 \overline{1}}$,

which is never zero by the positive definiteness of the metric. Any (real) closed 1 -form $\theta$ is given by $\theta=a \omega^{1}+\bar{a} \omega^{\overline{1}}+b\left(\omega^{2}+\omega^{\overline{2}}\right)$, where $a \in \mathbb{C}$ and $b \in \mathbb{R}$. Since the coefficients of the 3 -form $2 \theta \wedge F$ in $\omega^{13 \overline{3}}$ and $\omega^{23 \overline{3}}$ are equal to $i a t^{2}$ and $i b t^{2}$, respectively, it follows from (9) and the positive definiteness of the metric that the condition $\partial F=\theta^{1,0} \wedge F$ is satisfied if and only if $a=b=0$. However, this contradicts $\partial F \neq 0$, so the manifold $\left(\mathcal{N}_{1}, \mathcal{I}_{1}^{\rho}\right)$ has no locally conformal Kähler metrics for $\rho=0,1$. 
The nonexistence of SKT metrics follows directly from

$$
2 \partial \bar{\partial} F=-i t^{2}\left(\rho^{2}+(1-\rho)^{2}\right) \omega^{12 \overline{1} \overline{2}} \neq 0 .
$$

Finally, it is straightforward to see that

$$
4 \partial F \wedge F=(1-\rho)\left(i t^{2} u+\bar{v} z\right) \omega^{123 \overline{1} \overline{2}}-\left(s^{2} t^{2}-|v|^{2}\right) \omega^{123 \overline{1} \overline{3}}
$$

and $\bar{\partial}\left(\bigwedge^{3,1}\right)=\left\langle\rho \omega^{123 \overline{1} \overline{2}}\right\rangle$. Therefore, $\partial F^{2}$ cannot be $\bar{\partial}$-exact, and we conclude that the manifold $\left(\mathcal{N}_{1}, \mathcal{I}_{1}^{\rho}\right)$ is not strongly Gauduchon for $\rho=0,1$.

Corollary 5.2. For compact complex manifolds, the $\mathcal{C}^{\infty}$-pure-and-full property is unrelated to the existence of SKT, locally conformal Kähler, Vaisman, balanced or strongly Gauduchon metrics.

Proof. In view of Theorem 2.3 and Proposition 5.1, it suffices to show the existence of SKT, Vaisman (hence locally conformal Kähler) and balanced (hence strongly Gauduchon) nilmanifolds that do not satisfy the $\mathcal{C}^{\infty}$-pure-and-full property, that is, that are not isomorphic to $\left(\mathcal{N}_{\epsilon}, \mathcal{I}_{\epsilon}^{\rho}\right), \epsilon, \rho \in\{0,1\}$. Again, the existence of such nilmanifolds can be derived from the classification results in $[9,14,26$, but for the sake of completeness, we here provide an explicit example of each type.

First, let us consider the nilmanifold $\mathcal{N}_{0}$ endowed with the invariant complex structure defined by

$$
d \omega^{1}=d \omega^{2}=0, \quad d \omega^{3}=\omega^{12}+\omega^{1 \overline{1}}+\frac{1}{2} \omega^{2 \overline{2}},
$$

i.e. defined by the complex equations (I) for $\rho=1, \lambda=0$ and $D=\frac{1}{2}$. It is clear that the Hermitian metric $F=\frac{i}{2}\left(\omega^{1 \overline{1}}+\omega^{2 \overline{2}}+\omega^{3 \overline{3}}\right)$ satisfies the SKT condition, so we get a compact complex manifold that is SKT but not $\mathcal{C}^{\infty}$-pure-and-full.

Now, let us consider the nilmanifold $M$ whose underlying Lie algebra is $\mathfrak{h}_{3}$ in the notation of [9], endowed with the invariant complex structure defined by the equations (I) for $\rho=\lambda=0$ and $D=1$, that is,

$$
d \omega^{1}=d \omega^{2}=0, \quad d \omega^{3}=\omega^{1 \overline{1}}+\omega^{2 \overline{2}} .
$$

The Hermitian metric $F=\frac{i}{2}\left(\omega^{1 \overline{1}}+\omega^{2 \overline{2}}+\omega^{3 \overline{3}}\right)$ is a locally conformal Kähler metric whose Lee form $\theta=\omega^{3}+\omega^{\overline{3}}$ is parallel. Hence, one has an example of a compact Vaisman manifold which does not satisfy the $\mathcal{C}^{\infty}$-pure-and-full property.

Finally, let us consider the nilmanifold $\mathcal{N}_{0}$ endowed with the invariant complex structure defined by

$$
d \omega^{1}=d \omega^{2}=0, \quad d \omega^{3}=\omega^{12}+\omega^{1 \overline{1}}-\frac{1}{8} \omega^{2 \overline{2}} .
$$

i.e. defined by the complex equations (I) for $\rho=1, \lambda=0$ and $D=-\frac{1}{8}$. The Hermitian metric $F=4 i \omega^{1 \overline{1}}+\frac{i}{2}\left(\omega^{2 \overline{2}}+\omega^{3 \overline{3}}\right)$ satisfies the balanced condition, so we get a compact balanced manifold that does not satisfy the $\mathcal{C}^{\infty}$-pure-and-full property.

Another interesting aspect is the existence of some relation between the behaviour of the Frölicher spectral sequence $\left\{E_{r}\right\}_{r \geq 1}$ and the $\mathcal{C}^{\infty}$-pure-and-fullness of compact complex manifolds. It is well-known that if the Frölicher spectral sequence of $X$ degenerates at the first step and there is a weight 2 formal Hodge decomposi- 
tion, then $X$ is $\mathcal{C}^{\infty}$-pure-and-full (see [5, 12, 19]). Note that by [9, Theorem 4.1] we have that $E_{1}\left(\mathcal{N}_{\epsilon}, \mathcal{I}_{\epsilon}^{\rho}\right) \not E_{2}\left(\mathcal{N}_{\epsilon}, \mathcal{I}_{\epsilon}^{\rho}\right) \cong E_{\infty}\left(\mathcal{N}_{\epsilon}, \mathcal{I}_{\epsilon}^{\rho}\right)$ for $\epsilon, \rho \in\{0,1\}$. Furthermore:

Proposition 5.3. For compact complex manifolds, the $\mathcal{C}^{\infty}$-pure-and-full property and the degeneration at $E_{1}$ of the Frölicher spectral sequence are unrelated. Moreover, there exists a compact complex manifold $X$ with $E_{1}(X) \cong E_{\infty}(X)$ whose Hodge numbers satisfy the symmetry $h_{\bar{\partial}}^{p, q}(X)=h_{\bar{\partial}}^{q, p}(X)$ for every $p, q \in \mathbb{N}$, which is neither $\mathcal{C}^{\infty}$-pure nor $\mathcal{C}^{\infty}$-full.

Proof. Let us consider the complex nilmanifold $X$ defined by the complex structure equations in the family (I) for $\rho=\lambda=1$ and $D=0$, i.e.

$$
d \omega^{1}=d \omega^{2}=0, \quad d \omega^{3}=\omega^{12}+\omega^{1 \overline{1}}+\omega^{1 \overline{2}} .
$$

By [9, Proposition 4.3], $X$ has Frölicher spectral sequence degenerating at $E_{1}$, and its Hodge numbers satisfy $h_{\bar{\partial}}^{p, q}(X)=h_{\bar{\partial}}^{q, p}(X)$ for every $p, q \in \mathbb{N}$. However, $X$ is not $\mathcal{C}^{\infty}$-pure because $\left[i\left(\omega^{12}-\omega^{\overline{1} \overline{2}}\right)\right]=-2\left[i \omega^{1 \overline{1}}\right]-\left[i\left(\omega^{1 \overline{2}}+\omega^{2 \overline{1}}\right)\right]$ is a non-zero de Rham cohomology class that belongs to $H^{+}(X) \cap H^{-}(X)$. On the other hand, one can check that the cohomology class $\left[\omega^{23}+\omega^{2 \overline{3}}-\omega^{3 \overline{2}}+\omega^{\overline{2}} \overline{3}\right]$ is a non-zero class in $H_{\mathrm{dR}}^{2}(X ; \mathbb{R})$ which does not belong to $H^{+}(X)+H^{-}(X)$.

It seems to be unknown whether there is some general relation between the $\mathcal{C}^{\infty}$ pure-and-full property and the degeneration of the Frölicher sequence at some step greater than 1. As we noticed above, the Frölicher sequence of the $\mathcal{C}^{\infty}$-pure-and-full complex nilmanifolds $\left(\mathcal{N}_{\epsilon}, \mathcal{I}_{\epsilon}^{\rho}\right), \epsilon, \rho \in\{0,1\}$, satisfies $E_{1} \not E_{2} \cong E_{\infty}$. Note that this can indeed be seen as a restriction, because there also exist complex nilmanifolds whose Frölicher sequence has $E_{2}$ non-isomorphic to $E_{\infty}$ (see [9]). The following natural question arises:

Question 1. Does the $\mathcal{C}^{\infty}$-pure-and-full property of compact complex manifolds imply the degeneration of the Frölicher spectral sequence at the second step?

New complex invariants defined in terms of the Bott-Chern cohomology have been introduced by Angella and Tomassini in [7, which allows us to provide a characterization to the $\partial \bar{\partial}$-Lemma condition. If $n$ is the complex dimension of $X=$ $(M, J)$, let $\Delta^{k}(M, J)=\sum_{p+q=k}\left(h_{\mathrm{BC}}^{p, q}(M, J)+h_{\mathrm{BC}}^{n-p, n-q}(M, J)\right)-2 b_{k}(M)$, where $h_{\mathrm{BC}}^{p, q}(M, J)$ denotes the dimension of the Bott-Chern cohomology group $H_{\mathrm{BC}}^{p, q}(M, J)$ and $b_{k}(M)$ the $k$-th Betti number of $M$. The complex invariants $\Delta^{k}(M, J)$ are non-negative, and $\Delta^{k}(M, J)=0$ for all $0 \leq k \leq 2 n$ if and only if $(M, J)$ satisfies the $\partial \bar{\partial}$-Lemma [7. If we denote $\Delta(M, J)=\sum_{k=1}^{2 n} \Delta^{k}(M, J)$, one has that compact complex manifolds $(M, J)$ satisfying the $\partial \bar{\partial}$-Lemma are characterized as those compact complex manifolds for which $\Delta(M, J)=0$.

Since every compact 4 -dimensional almost-complex manifold is $\mathcal{C}^{\infty}$-pure-and-full [12, we will now focus on the higher dimensional cases, i.e. real dimension greater than or equal to 6 . As the $\partial \bar{\partial}$-Lemma (equivalently, the vanishing of the complex invariant $\Delta$ ) implies $\mathcal{C}^{\infty}$-pure-and-fullness, one might expect to obtain low values of $\Delta$ for those compact complex manifolds satisfying the $\mathcal{C}^{\infty}$-pure-and-full property. Surprisingly, the propositions below suggest a possible relation between the $\mathcal{C}^{\infty}$ pure-and-full property and the complex structures for which $\Delta$ attains a maximal value (at least for nilmanifolds).

Let $M$ be a nilmanifold of real dimension 6 admitting invariant complex structures $J$, and suppose that the Lie algebra underlying $M$ is not isomorphic to $\mathfrak{h}_{7}$, 
in the notation of 9 . Let $\mathcal{X}$ be the set of all these pairs $(M, J)$. The reason to exclude $\mathfrak{h}_{7}$ is that the invariant $\Delta$ is not known for any maximal lattice in the Lie group of $\mathfrak{h}_{7}$ (for more details see [3, 18] and the references therein).

Proposition 5.4. Let $M$ be a 6-dimensional nilmanifold endowed with an invariant complex structure $J$ such that the underlying Lie algebra of $M$ is not isomorphic to $\mathfrak{h}_{7}$. Let $\mathcal{X}$ be the set of all these pairs $(M, J)$. If $(\tilde{M}, \tilde{J}) \in \mathcal{X}$ is a pair such that $\Delta(\tilde{M}, \tilde{J}) \geq \Delta(M, J)$ for any $(M, J) \in \mathcal{X}$, then $(\tilde{M}, \tilde{J})$ is $\mathcal{C}^{\infty}$-pure-and-full.

Proof. From [3, Table 2] and [18, Appendix 6] the invariant $\Delta(M, J)$ can be computed for any pair $(M, J)$. It turns out that the maximal value of $\Delta(M, J)$ when $(M, J)$ runs the space of all 6-nilmanifolds $M$ endowed with invariant complex structures $J$ is equal to 34 . Furthermore, $(\tilde{M}, \tilde{J})$ satisfies that $\Delta(\tilde{M}, \tilde{J})=34$ if and only if $\tilde{M}$ is precisely the nilmanifold $\mathcal{N}_{1}$ and $\tilde{J}$ is isomorphic either to the complex structure $\mathcal{I}_{1}^{0}$ or to the complex structure $\mathcal{I}_{1}^{1}$ given in Theorem 2.3 . Therefore, $(\tilde{M}, \tilde{J})$ is $\mathcal{C}^{\infty}$-pure-and-full.

Recall that the minimal value of $\Delta(M, J)$ when $(M, J)$ runs the space of all nilmanifolds $M$ endowed with invariant complex structures $J$ is equal to 0 , and it is attained at the complex tori, which are the only $\partial \bar{\partial}$-nilmanifolds.

Proposition 5.5. Let $M$ be a nilmanifold, not a torus, of real dimension 6 admitting an invariant complex structure $\tilde{J}$ such that $(M, \tilde{J})$ is $\mathcal{C}^{\infty}$-pure-and-full. Then, $\Delta(M, J)$ attains a maximal value at $(M, \tilde{J})$ when $J$ runs the space of all the invariant complex structures on the nilmanifold $M$.

Proof. By Theorem 2.3 it suffices to look at the spaces of all invariant complex structures on the nilmanifolds $\mathcal{N}_{0}$ and $\mathcal{N}_{1}$. For $M=\mathcal{N}_{1}$ it is clear that $\Delta$ attains a maximal value at the complex structures $\mathcal{I}_{1}^{0}$ and $\mathcal{I}_{1}^{1}$, as a consequence of Proposition 5.4.

Let $J$ be any invariant complex structure on $M=\mathcal{N}_{0}$. From [3, Table 2] and [18, Appendix 6] we have that $\Delta\left(\mathcal{N}_{0}, J\right)$ attains its maximal value when $J$ runs the space of all the invariant complex structures on $\mathcal{N}_{0}$ (which is equal to 24) if and only if $J$ is isomorphic to $\mathcal{I}_{0}^{0}$ or $\mathcal{I}_{0}^{1}$.

These results seem to suggest the existence of some possible relationship between the $\mathcal{C}^{\infty}$-pure-and-full property and those pairs $(M, J)$ where the complex invariant $\Delta(M, J)$ attains maximal values.

Question 2. Do Propositions 5.4 and 5.5 hold in higher dimensions? Do they hold for a more general class of compact complex manifolds?

\section{ACKNOWLEDGMENTS}

This work has been partially supported by the projects MINECO (Spain), MTM2011-28326-C02-01 and MTM2014-58616-P, and Gobierno de Aragón/Fondo Social Europeo, grupo consolidado E15-Geometría. The first author is also supported by a DGA predoctoral scholarship. The authors wish to thank the referee for useful comments and suggestions. 


\section{REFERENCES}

[1] Daniele Angella, The cohomologies of the Iwasawa manifold and of its small deformations, J. Geom. Anal. 23 (2013), no. 3, 1355-1378, DOI 10.1007/s12220-011-9291-z. MR.3078358

[2] Daniele Angella, Cohomological aspects in complex non-Kähler geometry, Lecture Notes in Mathematics, vol. 2095, Springer, Cham, 2014. MR3137419

[3] Daniele Angella, Maria Giovanna Franzini, and Federico Alberto Rossi, Degree of nonKählerianity for 6-dimensional nilmanifolds, Manuscripta Math. 148 (2015), no. 1-2, 177211, DOI 10.1007/s00229-015-0734-x. MR3377754

[4] D. Angella and H. Kasuya, Cohomologies of deformations of solvmanifolds and closedness of some properties, to appear in Mathematica Universalis (episciences-math).

[5] Daniele Angella and Adriano Tomassini, On cohomological decomposition of almost-complex manifolds and deformations, J. Symplectic Geom. 9 (2011), no. 3, 403-428. MR2817781

[6] Daniele Angella and Adriano Tomassini, On the cohomology of almost-complex manifolds, Internat. J. Math. 23 (2012), no. 2, 1250019, 25, DOI 10.1142/S0129167X11007604. MR2890470

[7] Daniele Angella and Adriano Tomassini, On the $\partial \bar{\partial}$-lemma and Bott-Chern cohomology, Invent. Math. 192 (2013), no. 1, 71-81, DOI 10.1007/s00222-012-0406-3. MR3032326

[8] Daniele Angella, Adriano Tomassini, and Weiyi Zhang, On cohomological decomposability of almost-Kähler structures, Proc. Amer. Math. Soc. 142 (2014), no. 10, 3615-3630, DOI 10.1090/S0002-9939-2014-12049-1. MR3238437

[9] M. Ceballos, A. Otal, L. Ugarte, and R. Villacampa, Invariant complex structures on 6-nilmanifolds: classification, Frölicher spectral sequence and special Hermitian metrics, J. Geom. Anal. 26 (2016), no. 1, 252-286, DOI 10.1007/s12220-014-9548-4. MR3441513

[10] Pierre Deligne, Phillip Griffiths, John Morgan, and Dennis Sullivan, Real homotopy theory of Kähler manifolds, Invent. Math. 29 (1975), no. 3, 245-274. MR0382702

[11] S. K. Donaldson, Two-forms on four-manifolds and elliptic equations, inspired by S. S. Chern, Nankai Tracts Math., vol. 11, World Sci. Publ., Hackensack, NJ, 2006, pp. 153-172, DOI 10.1142/9789812772688_0007. MR2313334

[12] Tedi Draghici, Tian-Jun Li, and Weiyi Zhang, Symplectic forms and cohomology decomposition of almost complex four-manifolds, Int. Math. Res. Not. IMRN 1 (2010), 1-17, DOI 10.1093/imrn/rnp113. MR2576281

[13] Tedi Draghici, Tian-Jun Li, and Weiyi Zhang, On the J-anti-invariant cohomology of almost complex 4-manifolds, Q. J. Math. 64 (2013), no. 1, 83-111, DOI 10.1093/qmath/har034. MR3032090

[14] Nicola Enrietti, Anna Fino, and Luigi Vezzoni, Tamed symplectic forms and strong Kähler with torsion metrics, J. Symplectic Geom. 10 (2012), no. 2, 203-223. MR.2926995

[15] Anna Fino, Antonio Otal, and Luis Ugarte, Six-dimensional solvmanifolds with holomorphically trivial canonical bundle, Int. Math. Res. Not. IMRN 24 (2015), 13757-13799. MR 3436163

[16] Anna Fino and Adriano Tomassini, On some cohomological properties of almost complex manifolds, J. Geom. Anal. 20 (2010), no. 1, 107-131, DOI 10.1007/s12220-009-9098-3. MR2574723

[17] Richard Hind, Costantino Medori, and Adriano Tomassini, On taming and compatible symplectic forms, J. Geom. Anal. 25 (2015), no. 4, 2360-2374. MR3427128

[18] Adela Latorre, Luis Ugarte, and Raquel Villacampa, On the Bott-Chern cohomology and balanced Hermitian nilmanifolds, Internat. J. Math. 25 (2014), no. 6, 1450057, 24, DOI 10.1142/S0129167X14500578. MR3225581

[19] Tian-Jun Li and Weiyi Zhang, Comparing tamed and compatible symplectic cones and cohomological properties of almost complex manifolds, Comm. Anal. Geom. 17 (2009), no. 4, 651-683, DOI 10.4310/CAG.2009.v17.n4.a4. MR.2601348

[20] M. L. Michelsohn, On the existence of special metrics in complex geometry, Acta Math. 149 (1982), no. 3-4, 261-295, DOI 10.1007/BF02392356. MR688351

[21] Iku Nakamura, Complex parallelisable manifolds and their small deformations, J. Differential Geometry 10 (1975), 85-112. MR0393580

[22] Dan Popovici, Deformation limits of projective manifolds: Hodge numbers and strongly Gauduchon metrics, Invent. Math. 194 (2013), no. 3, 515-534, DOI 10.1007/s00222-0130449-0. MR.3127061 
[23] Dan Popovici, Deformation openness and closedness of various classes of compact complex manifolds; examples, Ann. Sc. Norm. Super. Pisa Cl. Sci. (5) 13 (2014), no. 2, 255-305. MR.3235516

[24] S. M. Salamon, Complex structures on nilpotent Lie algebras, J. Pure Appl. Algebra 157 (2001), no. 2-3, 311-333, DOI 10.1016/S0022-4049(00)00033-5. MR1812058

[25] Jeffrey Streets and Gang Tian, A parabolic flow of pluriclosed metrics, Int. Math. Res. Not. IMRN 16 (2010), 3101-3133, DOI 10.1093/imrn/rnp237. MR2673720

[26] Luis Ugarte, Hermitian structures on six-dimensional nilmanifolds, Transform. Groups 12 (2007), no. 1, 175-202, DOI 10.1007/s00031-005-1134-1. MR.2308035

[27] Chun-Chun Wu, On the geometry of superstrings with torsion, ProQuest LLC, Ann Arbor, MI. Thesis (Ph.D.)-Harvard University, 2006. MR 2708755

Departamento de Matemáticas-I.U.M.A., Universidad de Zaragoza, Campus Plaza SAN Francisco, 50009 Zaragoza, SPAIN

E-mail address: adela@unizar.es

Departamento de Matemáticas-I.U.M.A., Universidad de Zaragoza, Campus Plaza San Francisco, 50009 Zaragoza, Spain

E-mail address: ugarte@unizar.es 